Research

\title{
Prevalence of physical activity behaviour in older people: Findings from the Dynamic Analyses to Optimise Ageing (DYNOPTA) project and Australian national survey data
}

\author{
Jane Sims \\ Healthy Ageing Research Unit, School of Primary Health Care; and \\ Monash Institute of Health Services Research, Monash University, \\ Melbourne, Victoria, Australia

\section{Carole L Birrell} \\ Centre for Statistical and Survey Methodology, University of \\ Wollongong, Wollongong, New South Wales, Australia
}

\section{Susan Hunt and Colette Browning}

Healthy Ageing Research Unit, School of Primary Health Care; and Monash Institute of Health Services Research, Monash University, Melbourne, Victoria, Australia

\section{Richard A Burns}

Centre for Research on Ageing, Health and Wellbeing, Australian National University, Canberra, Australian Capital Territory, Australia

\section{Paul Mitchell}

Centre for Vision Research, Westmead Millennium Institute, University of Sydney, Sydney, New South Wales, Australia

Aim: Many older people lead sedentary lives. National Health Survey physical activity prevalence data provide limited coverage of the 'old old' ( $\geq 75$ years).

Method: The Dynamic Analyses to Optimise Ageing (DYNOPTA) project's dataset provided physical activity data for 13420 participants. Physical activity (walking, moderate- and vigorous-intensity activities in the previous week) was measured. Data were weighted and prevalence was calculated.

Results: The frequency of walking in DYNOPTA was similar to that in the national sample. Walking remained relatively stable until a decline among persons aged 80 years and over; moderate and vigorous activity declined in all but a minority of persons aged 70 years and over. Although DYNOPTA participants reported more physical activity than those in the contemporary national survey, the rates of sedentary behaviour were high.

Conclusion: We require more information about the 'oldest old' $(85+$ years). There is great scope for increasing physical activity, even walking, among older people.

Key words: oldest old, physical activity, prevalence.

\section{Introduction}

The proportion of Australians aged 65 years and older is set to increase to around $25 \%$ by 2056 [1]. Currently, those

Correspondence to: Dr Jane Sims, Monash University,

Faculty of Medicine, Nursing and Health Sciences.

Email:jane.sims@monash.edu.au aged 85 years and older comprise a small proportion of the population at $1.6 \%$, but this proportion is also set to increase to around $7.3 \%$ by 2056 [1]. Such changes in population demographics are expected worldwide. The World Health Organisation (WHO) has emphasised the need for national authorities to promote healthy ageing. The WHO has called on its members to 'optimise opportunities for health, participation and security in order to enhance quality of life as people age' [2; p. iv]. We know that protective factors for healthy ageing include engaging in social and productive activities, not smoking, moderate alcohol intake and some level of physical activity [3]. However, the prevalence of physical activity among the oldest Australians (75 years and over) is unclear, as typically, national databases group all older people into the category of ' 65 years and over' for reporting purposes. Such grouping covers people from age 65 through to centenarians. It is important to know the prevalence of physical activity across smaller age intervals in order to provide a complete picture of the physical activity undertaken by older people. The potential negative health outcomes brought about by sedentary behaviour [4] can be better assessed by using prevalence rates in specific age subgroups of the population, than by grouping all those aged ' 65 years and above'.

There has been limited international reporting of physical activity prevalence in the old old ( $\geq 75$ years) [5]. The Zutphen Elderly Study (men aged 65-84 at baseline) reported a stable 5 -year prevalence of sedentary behaviour of about $28 \%$ [6]. Total physical activity decreased for most participants across the 10-year follow-up period: mean total physical activity decreased by $33 \%$, equivalent to 28 minutes per day. In Australia, population-level physical activity data have been collected via National Health Surveys (NHS): while those $\geq 75$ years are included, their data are aggregated, limiting interpretation.

Two national physical activity surveys were conducted in 1997 and 1999 [7,8]. According to the 1999 data [8], less than half of Australians aged over 65 years performed 'sufficient physical activity to produce health benefit', defined as the accumulation of at least 150 minutes of moderate and/or vigorous activity per week [8]. Further, as many as one-third of those 'insufficiently active' were completely sedentary [9]. The absolute prevalence of sedentary behaviour increased with age, and women were generally less active than men. Among older people with English as a second language and those with chronic conditions, physical activity rates 
are lower than in the general population [10]. Given the risk of health impacts associated with inactivity, there is much to gain at both an individual and societal level from older people being physically active. Interestingly, the national decline in physical activity levels in Australian adults from 1997 to 1999 was not observed among older adults. For those aged 60-75 years, physical activity (mean time spent in walking, other moderate activity, vigorous gardening and yardwork, and other vigorous activities) increased between 1997 and 1999. Walking was frequent in both men and women, across age groups, and increased in prevalence among older men. In 1999, older adults of both sexes spent the greatest time in moderate-intensity physical activities. Men spent more time than women doing vigorous-intensity gardening and yardwork, and this contrast increased with age. Overall, $17.9 \%$ were sedentary, $28.1 \%$ did insufficient activity and the remaining $54.1 \%$ performed sufficient activity (based on sufficient time, defined as 150 minutes of activity, summating walking, moderate and $2 \mathrm{x}$ vigorous). Slightly fewer $(43.6 \%)$ were sufficiently active using a more rigorous definition (i.e. at least 150 minutes of activity over at least five sessions per week). While 'usual' participation (i.e. over the past 6 months) in 'sufficient' activity declined with age for men until age 60, it increased to levels greater than observed among younger men (30-44 years). For women, 'usual' participation at 'sufficient' levels declined across age groups. These data are encouraging, particularly given media campaigns targeted at older persons in 1998-1999 [9,11]. However interpretation is limited by the broad age grouping and the sample's age cut-off of 75 years.

As noted above, a limitation of the NHS is that they do not provide detailed information on the 'old old'. The Dynamic Analyses to Optimise Ageing (DYNOPTA) project [12] includes physical activity data from older people aged up to 106 and the current study aimed to examine the physical activity behaviour of the 'young old' and 'old old' to explore more fully the physical activity behaviour of older Australians using DYNOPTA data. This paper discusses the prevalence of physical activity in older Australians using national and research data that allow a more detailed analysis of physical activity prevalence within this heterogeneous age group. We also consider the implications of the data for future 'healthy ageing' interventions.

The present analysis addresses the following questions:

- What are the physical activity patterns of the 'old old'?

- Does physical activity behaviour alter with advancing age and are there sex differences?

- Do physical activity trends in DYNOPTA reflect those reported in Australian national datasets?

\section{Methods}

\section{DYNOPTA}

The broad aims of DYNOPTA are to identify effective pathways to compress morbidity and optimise ageing [12].
DYNOPTA is a pooled dataset comprising information from nine Australian Longitudinal Studies of Ageing. Data have been harmonised from the contributing studies to produce new variables and constructs derived from complex harmonisation procedures. Where possible, variables were harmonised to enable comparison with Australian benchmarks. The physical activity data were harmonised to derive measures reflecting the recommended level of physical activity per week for adults [13].

The research program focuses on four outcomes that contribute greatly to the burden of disease and disability, namely dementia and cognition, mental health, sensory impairment and mobility/activity limitations. Further details of the project have been published elsewhere [12].

\section{Participants}

Participants were selected from those studies where physical activity was measured. We chose data waves (data collection periods) to maximise the number of respondents while minimising the timeframe over which the included studies' data were collected. For this paper, data were available from four studies for women and three for men. Wave 2 data were used from three studies: Blue Mountains Eyes Study (BMES); Australian Longitudinal Study of Women's Health old cohort (ALSWH); and Sydney Older Persons Study (SOPS), and wave 1 data from the fourth (Australian Diabetes, Obesity and Lifestyle Study (AusDiab)). Wave 2's time period covered 19972000, with the exception of one dataset (SOPS), which covered 1994-1996. The participants were grouped into four age categories: $65-69,70-74,75-79$ and $80+$ years. The latter category was aggregated given the diminishing numbers.

The 2001 NHS was chosen for comparison, being the chronologically closest national dataset. The 2001 NHS was conducted in 17918 private dwellings selected from nonsparsely settled areas of Australia [14]. The sample design ensured that within each State or Territory each person had an equal chance of selection. Information was obtained about an adult, all children aged 0-6 years and one child aged 7-17 years in each selected household. A total of 26863 persons fully responded to the survey. Trained Australian Bureau of Statistics (ABS) interviewers personally interviewed the selected adult member of the household. The sample included 3198 Aboriginal and Torres Strait Islander adults and children. Data are available in 5-year age bands up to age 74 . Beyond that, aggregated data are given for persons aged $\geq 75$ years.

\section{Measures}

\section{Harmonisation}

Detailed information about the overall harmonisation process of DYNOPTA is provided elsewhere [12]. The composite studies for the current analyses used items similar to the Active Australia Survey regarding the number of activity sessions and the time spent doing these activities. The Active Australia 
Survey references to the past week. Only walking, moderateand vigorous-intensity leisure activities were included in the harmonisation process. Household chores (e.g. 'yardwork') and gardening were not included. One study (SOPS) asked only about walking frequency.

\section{Frequency}

The definition of a session varied across studies. Two of the datasets specified a session as activity for 10 minutes or more, while the remainder did not specify a time. To account for outliers or coding errors, the upper number of sessions was capped at 42 . (This was based on a feasible assumption of six 10-minute sessions per day, adding to 42 per week.) Six categories were created: none, $1-2,3-4,5-7,8-14$ and $\geq 15$ sessions per week.

\section{Duration}

Three studies reported the total time spent in activity: walking, moderate and vigorous activity. Values in hours were converted to minutes per week $(\mathrm{m} / \mathrm{w})$. To account for outliers or coding errors, the total was capped at $1680 \mathrm{~m} / \mathrm{w}$, in line with the international protocol used for this measure by the International Physical Activity Questionnaire [15].

\section{National Health Survey}

The 2001 NHS asked about physical exercise undertaken for recreation, sport or health/fitness purposes. The topic excluded physical activity undertaken in the course of work and activity undertaken for reasons other than recreation, sport or health/fitness. All respondents aged 15 years or above were asked whether, during the previous 2 weeks, they did any: walking for sport, recreation or fitness; moderate exercise (apart from walking); or vigorous exercise. For each of these categories, they were asked (i) the number of times they had done that exercise in the previous 2 weeks; and (ii) the total amount of time spent (hours and minutes) doing that exercise over that 2 weeks. Moderate exercise was defined as exercise that caused a moderate increase in the heart rate or breathing of the respondent. Vigorous exercise was defined as exercise that caused a large increase in the respondent's heart rate or breathing. For each activity type, a score was obtained by multiplying frequency by duration of exercise and weighting by a metabolic equivalent of task (MET)-based intensity constant. A person was categorised as 'sedentary' if they scored less than 100. For example, a sedentary person could have reported walking once per week for 20 minutes, producing a MET score of 75 .

\section{Analyses}

The studies contributing to DYNOPTA used a variety of survey designs and differed in their geographic and demographic coverage. To produce population estimates from a sample survey, weights are usually calculated to account for differences in probabilities of selection and to ensure consistency with external information about the population, known as population benchmarks. Weights existed for wave 1 for one of the four contributing studies. For the remaining three studies, study weights at wave 1 were developed to adjust the sample in each study to the specific population from which it was drawn and to account for the probability of selection of each individual. These wave 1 weights were then adjusted to agree with the Estimated Resident Population for the relevant year, sex, age group and geographical area for each study.

To obtain study weights at wave 2 , wave 1 weights were adjusted to account for missing cases due to death or attrition. A logistic regression model for attrition used the following variables from wave 1 (where applicable): sex, partner status, education, age (binary variable if $\geq 75$ years), English as first language or English used at home, self-rated health and smoking status.

In order to provide estimates for a similar period of time, wave 2 data for ALSWH (1999), BMES (1997-1999) and SOPS (1994-1996) were combined with wave 1 data from AusDiab (1999-2000). Although the SOPS data covered an earlier period, it consisted of only $3 \%$ of the combined data for only one variable. To enable analysis of a pooled dataset consisting of a combination of studies and waves, final combined weights were calculated. The final weights combined the study specific weights according to their contributing sample sizes to the pooled dataset. For one variable (number of walking sessions), four studies (ALSWH, AusDiab, BMES and SOPS) contributed to the pooled data, and all except SOPS contributed for the remaining variables. Final weights were then benchmarked to the 1999 Estimated Resident Population at the sex by age group and major statistical region. Using the combined weights in the estimation procedure adjusts for the unequal probabilities of selection in the studies and allows for the contribution of the individual study to the pooled dataset. The final weights allow for any overlap of the studies at the geographic level.

The results reported for the estimated population means and proportions were determined using the final combined weights for the appropriate pooled dataset. The estimated standard errors for the prevalence estimates take into account the use of the final combined weights and the complex survey design for each study. Given the skew towards sedentary behaviour, weighted medians are presented. Age and sex comparisons were explored in the subsamples who reported some activity in each of the three modalities: walking, moderate activity and vigorous activity.

To determine whether there were significant or systematic differences in physical activity patterns between DYNOPTA and NHS, the DYNOPTA data were re-grouped to create an age group category '75+', to allow direct comparison of the means. The NHS activity variables were divided by two to produce a weekly physical activity variable for each exercise modality. Those reporting no activity (responding $0 \mathrm{~m} / \mathrm{w}$ ) were excluded. The data were then corrected for skewness using a natural logarithm transformation. Weighted analysis was performed using Stata version 11 (Stata Corp., College Station, TX, USA). 


\section{Results}

\section{Physical activity in DYNOPTA}

The prevalence of physical activity behaviour in DYNOPTA is presented in Table $1 \mathrm{a}-\mathrm{c}$ for walking, moderate- and vigorousintensity activity respectively. Women and people in the oldest age group were the most sedentary. The findings for each physical activity modality are described below, followed by comparison with NHS data.

\section{Walking}

About a quarter of men and over a third of women reported no walking for leisure. In terms of number of sessions walked per week, very few reported walking eight or more sessions per week (Table 1a). On average, men walked significantly more often than women $(t=2.1$, d.f. $10670, P=0.03)$ and there was a significant age-sex interaction effect $(t=2.6$, d.f. $10670, P=0.009)$. The median time spent walking was $180 \mathrm{~m} / \mathrm{w}$ in the youngest men (65-69), decreasing to $150 \mathrm{~m} / \mathrm{w}$ in the $70-74$ year olds and $120 \mathrm{~m} / \mathrm{w}$ in the two oldest groups. Men aged $80+$ walked the least. For women, the median was $90 \mathrm{~m} / \mathrm{w}$ in the youngest and oldest groups, $60 \mathrm{~m} / \mathrm{w}$ in the 70-74 year olds and $30 \mathrm{~m} / \mathrm{w}$ in the 75-79 year olds. Based on a recommended 30 minutes of activity on most days, men's walking time met the national physical activity recommendation, but the women's did not.

Figure 1a shows the mean time spent walking in the subsample where some walking was undertaken; men aged $80+$ years walked the least time. For women, there was no clear age trend.

\section{Moderate and vigorous activity}

Regular moderate and vigorous activity were even less prevalent than walking (Table 1). The median time men engaged in moderate activity was $240 \mathrm{~m} / \mathrm{w}$, apart from those 75-79 years, where it was $130 \mathrm{~m} / \mathrm{w}$. The median for women was $180 \mathrm{~m} / \mathrm{w}$ in the youngest and oldest groups, but zero in the other two age groups. As shown in Figure 1b, men tended to be more active than women, except for the 75-79 years age group. The women's average remained steady until a decline in the oldest group (80+ years).

Figure 1c shows the mean weekly figures among those reporting some vigorous activity, with a general trend for the means to decrease with age.

Comparing mean physical activity trends with national data The DYNOPTA mean physical activity patterns were compared with those in the 2001 NHS. The following findings focus on the people who reported some activity. To enable direct comparison, DYNOPTA data were re-grouped to create a ' $75+$ ' age group category, and NHS activity variables were expressed in terms of weekly physical activity for each exercise modality. Weighted means for individuals reporting some activity are shown in Table 2 . Analyses of the transformed mean differences are shown in Figure 2.

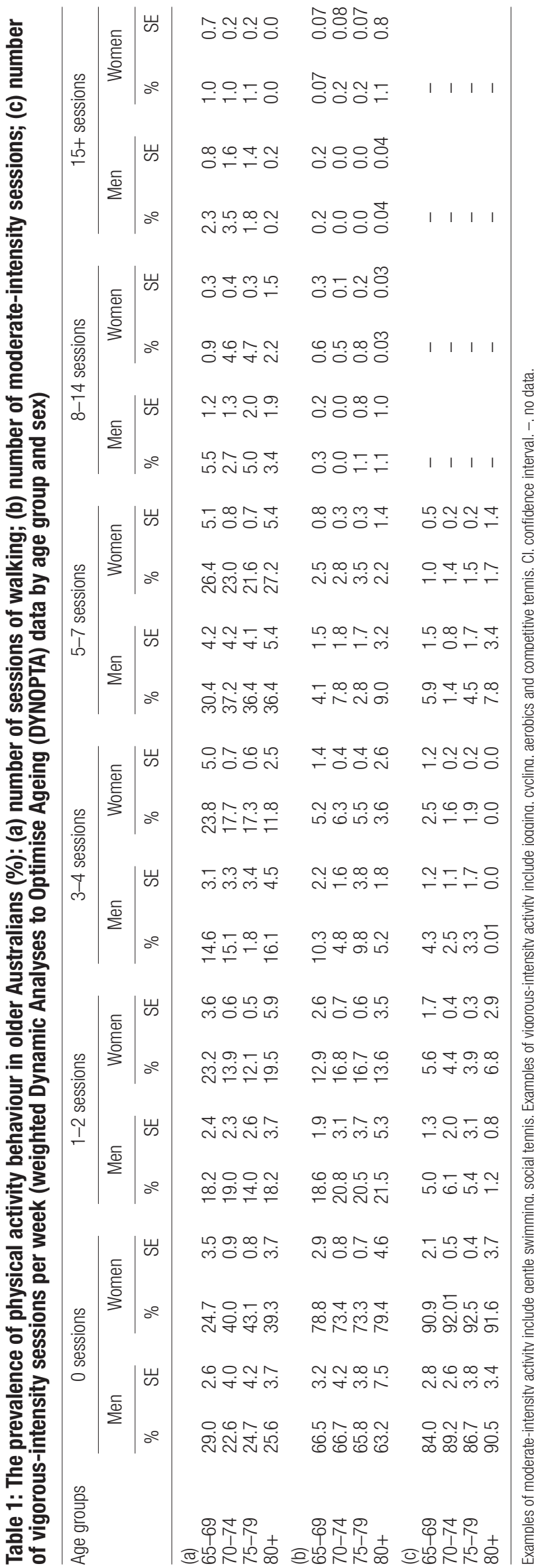


Figure 1: Mean time spent in (a) walking, (b) moderate activity and (c) vigorous activity by age and sex, Dynamic Analyses to Optimise Ageing (DYNOPTA) (excluding sedentary people).

(a)

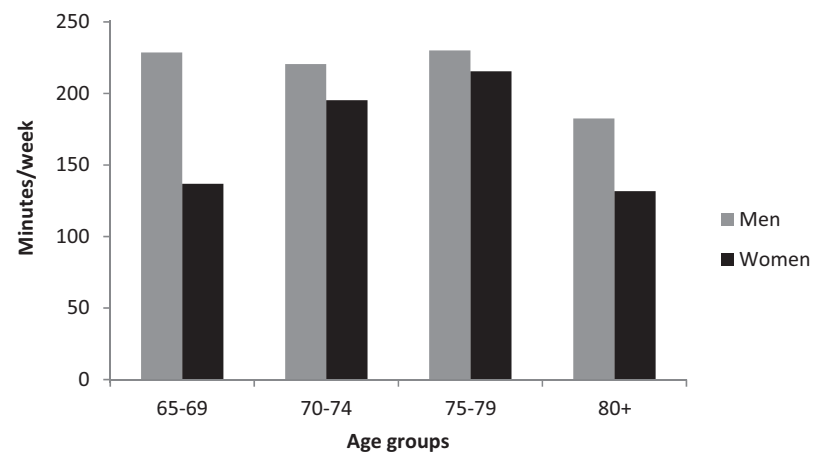

(b)

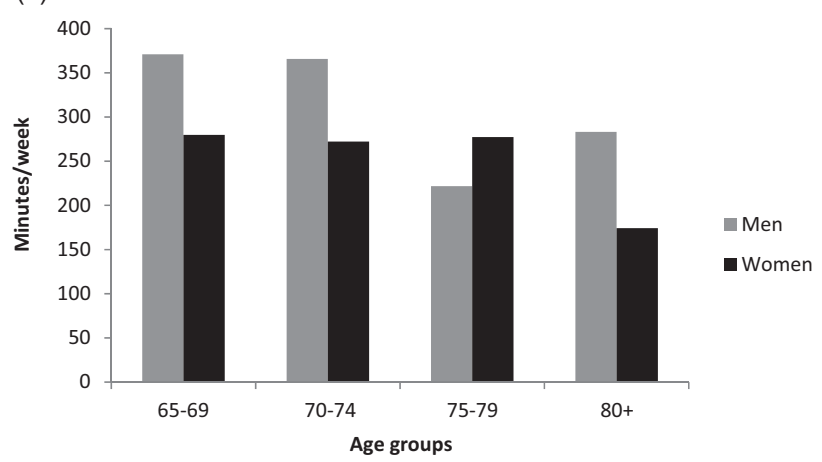

(c)

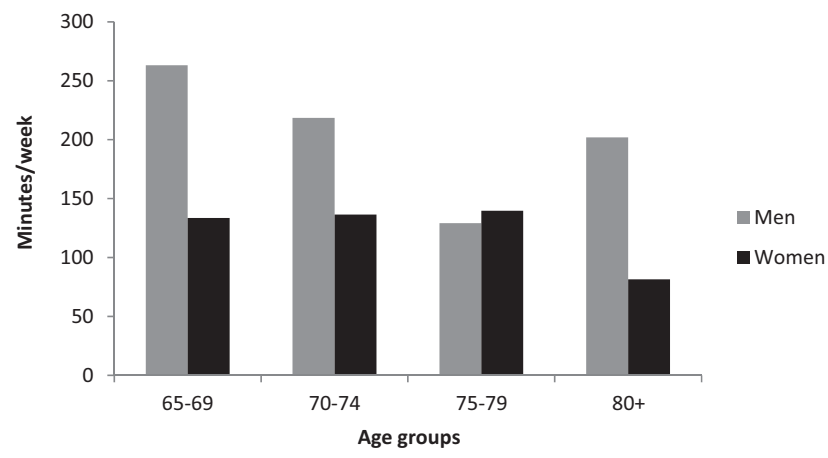

Fewer people were active in the 2001 NHS. As shown in Figure $2 \mathrm{a}$, the mean time spent walking was similar across the two datasets, across age groups and for both sexes. The exception was for the youngest DYNOPTA women, who did significantly less walking than their NHS counterparts $(t=2.4$, d.f. $49.3, P=0.02)$.

With regard to moderate activity, significant mean differences were seen (Figure 2b). The younger DYNOPTA men reported significantly more time doing moderate activity than their NHS counterparts (65-69 years, $t=3.2$, d.f. $39.4, P=0.003$; 70-74 years; $t=4.3$, d.f. 51.4, $P=0.0001)$. DYNOPTA women reported doing more moderate activity than their NHS counterparts, the mean difference being significant in the two older age groups $(70-74$ years; $t=4.5$, d.f. 31.8 , $P=0.0001,75+$ years; $t=2.2$, d.f. $46.6, P=0.03)$.
There was no significant difference in the vigorous activity patterns across the two datasets (Figure 2c). DYNOPTA men tended to report more vigorous activity, but the results require cautious interpretation because of the very small numbers.

\section{Discussion}

The findings from DYNOPTA data confirm the low physical activity prevalences among older Australians. These physical activity prevalences closely reflect the age and sex trends reported in national survey data, but the DYNOPTA data shed light on the duration of activity among those who were active.

Physical activity prevalence

The prevalence of physical activity behaviour in those aged 65 years and over was greater in men than in women. Time spent walking decreased with age in men; women reported less walking time than men across all age groups. A similar pattern was observed for moderate and vigorous activity. The DYNOPTA data enabled us to explore in detail patterns across the 'old old' (those aged 75 years and over). The greater detail about the 'old old' afforded by the data showed that men walk less as they get older and the decline in walking activity is most noticeable in the over $80 \mathrm{~s}$. Moderate activity behaviour declined with age, but the decline was most pronounced in the oldest women. A similar pattern was observed for vigorous activity, although a small number of the 'old old', particularly men, were still active at these intensity levels.

One reason for the decline in physical activity, even walking, could be an age-associated decline in physical mobility. Wave 1 harmonised data from the complete DYNOPTA population highlighted a marked decline in the ability to walk various distances (e.g. $1 \mathrm{~km}, 500 \mathrm{~m}, 100 \mathrm{~m}$ ), to mobilise within the home, and to transfer [16]. For example, while most people in their 70 s (80\% women and $83 \%$ men) were able to walk $1 \mathrm{~km}$ (with or without some difficulty), as these people aged the distance that could be walked decreased. The proportions who were more seriously disabled in mobilising and transferring also increased.

The prevalence of physical activity was greater in DYNOPTA than in the NHS dataset across age and sex groups. This finding indicates that older people may be more physically active than the national data suggest. As DYNOPTA is representative of the population, this larger sample of older people offers a more encouraging picture of the physical activity behaviour of older people.

However, looking at the findings in greater detail highlights that there is much scope for increasing levels of physical activity. For example, the youngest DYNOPTA women reported less time spent walking than their NHS counterparts. Targeting this age group to promote incidental activity such as walking has the potential to increase physical activity 
Table 2: Mean time spent performing physical activity: walking, moderate and vigorous activity, Dynamic Analyses to Optimise Ageing (DYNOPTA) and National Health Surveys (NHS) 2001 (non-sedentary respondents)

\begin{tabular}{|c|c|c|c|c|c|c|c|c|c|c|c|c|c|}
\hline \multirow[t]{2}{*}{ Sex } & \multirow[t]{2}{*}{$\begin{array}{l}\text { Age } \\
\text { groups }\end{array}$} & \multicolumn{6}{|c|}{$\begin{array}{c}\text { DYNOPTA (minutes/week) } \\
\text { (Cases reporting }>0 \text { minutes/week) }\end{array}$} & \multicolumn{6}{|c|}{$\begin{array}{c}\text { NHS (minutes/week) } \\
\text { (Cases reporting }>0 \text { minutes/week) }\end{array}$} \\
\hline & & Mean & SE & Lower Cl & Upper Cl & $n>0(\%)$ & Total $n$ & Mean & SE & Lower Cl & Upper Cl & $n>0(\%)$ & Total $n$ \\
\hline \multicolumn{14}{|c|}{ Walking } \\
\hline M & $65-69$ & 228.7 & 13.6 & 202.0 & 255.4 & 386 (89) & 430 & 231.1 & 17.0 & 196.3 & 266.0 & $235(56)$ & 414 \\
\hline M & $70-74$ & 220.6 & 23.9 & 173.7 & 267.4 & $372(87)$ & 427 & 255.3 & 15.3 & 223.9 & 286.6 & 239 (58) & 409 \\
\hline M & $75+$ & 207.9 & 11.8 & 184.7 & 231.1 & 416 (85) & 485 & 210.2 & 10.7 & 188.3 & 232.0 & 252 (46) & 544 \\
\hline W & 65-69 & 136.9 & 15.8 & 106.0 & 167.8 & 466 (89) & 519 & 183.5 & 15.0 & 152.8 & 214.2 & $267(56)$ & 469 \\
\hline W & $70-74$ & 195.3 & 5.2 & 185.2 & 205.5 & 2858 (62) & 4566 & 194.1 & 13.3 & 167.0 & 221.3 & 270 (52) & 513 \\
\hline W & $75+$ & 158.7 & 14.1 & 131.1 & 186.2 & $\begin{array}{l}3365(58) \\
7863\end{array}$ & $\begin{array}{r}5767 \\
12194\end{array}$ & 162.1 & 9.8 & 142.2 & 182.1 & $\begin{array}{l}367(39) \\
1630\end{array}$ & $\begin{array}{r}928 \\
3277\end{array}$ \\
\hline \multicolumn{14}{|c|}{ Moderate activity } \\
\hline M & $65-69$ & 371.0 & 36.7 & 299.0 & 443.1 & $214(77)$ & 277 & 243.1 & 28.0 & 185.7 & 300.4 & 135 (32) & 414 \\
\hline M & $70-74$ & 365.8 & 40.5 & 286.3 & 445.2 & $190(66)$ & 287 & 222.2 & 25.9 & 169.3 & 275.1 & $130(31)$ & 409 \\
\hline M & $75+$ & 250.5 & 31.3 & 189.2 & 311.9 & 233 (67) & 346 & 253.6 & 31.2 & 189.8 & 317.4 & 128 (23) & 544 \\
\hline W & $65-69$ & 279.8 & 49.7 & 182.4 & 377.2 & $229(71)$ & 320 & 166.4 & 15.9 & 133.9 & 198.8 & 137 (29) & 469 \\
\hline W & $70-74$ & 272.2 & 8.1 & 256.2 & 288.2 & $1341(30)$ & 4411 & 223.8 & 25.5 & 171.6 & 276.0 & $120(23)$ & 513 \\
\hline W & $75+$ & 193.2 & 13.4 & 167.0 & 219.5 & $\begin{array}{l}1666(29) \\
3873\end{array}$ & $\begin{array}{r}5629 \\
11270\end{array}$ & 196.3 & 20.2 & 155.0 & 237.5 & $\begin{array}{l}153(16) \\
803\end{array}$ & $\begin{array}{r}928 \\
3277\end{array}$ \\
\hline \multicolumn{14}{|c|}{ Vigorous activity } \\
\hline M & 65-69 & 263.2 & 72.4 & 121.2 & 405.2 & $152(73)$ & 207 & 96.6 & 25.8 & 43.9 & 149.3 & $20(4)$ & 414 \\
\hline M & 70-74 & 218.5 & 53.2 & 114.3 & 322.8 & 126 (58) & 215 & 222.9 & 78.2 & 62.9 & 382.9 & 17 (4) & 409 \\
\hline M & $75+$ & 163.1 & 35.4 & 93.8 & 232.4 & 149 (58) & 254 & 135.9 & 49.4 & 34.8 & 237.0 & 9 (1) & 544 \\
\hline W & 65-69 & 133.6 & 17.0 & 100.2 & 166.9 & 171 (62) & 272 & 270.1 & 110.4 & 44.3 & 495.8 & $13(2)$ & 469 \\
\hline W & $70-74$ & 136.4 & 10.9 & 115.0 & 157.8 & $474(10)$ & 4362 & 135.7 & 34.1 & 66.0 & 205.5 & $13(2)$ & 513 \\
\hline W & $75+$ & 85.1 & 20.8 & 44.3 & 125.9 & $\begin{array}{l}577(10) \\
1649\end{array}$ & $\begin{array}{r}5566 \\
10876\end{array}$ & 104.2 & 52.0 & -2.2 & 210.6 & $\begin{array}{l}11(1) \\
83\end{array}$ & $\begin{array}{r}928 \\
3277\end{array}$ \\
\hline
\end{tabular}

Very small sample sizes for doing any vigorous activity in NHS dataset. Cl, confidence interval; M, men; W, women.

behaviour as they enter their 70 s and 80 s. While the women in their 70 s and beyond reported more moderate activity than their NHS counterparts, the proportion who did any moderate activity was much lower than for the men. There is potential to encourage more moderate activities in these women. The DYNOPTA data show that some vigorous activity does occur in both men and women across the age groups, possibly as people continue the activities they have done throughout their adulthood.

The findings were not adjusted for health status. An explanation of the differences could be that the DYNOPTA sample is a healthier sample than the older adults in the NHS. Previous comparison of the disease prevalence among 12000 of the DYNOPTA dataset for 2000-2002 with the 2001 NHS data indicated that this is not necessarily the case. While diabetes prevalence was lower, asthma and hypertension rates were higher in DYNOPTA than the NHS [17]. Analyses of depression prevalence [18] and dementia prevalence [19] in DYNOPTA do not suggest a healthy cohort effect. Bartsch and colleagues [20] recently reported that Short Form (SF-36) scores in DYNOPTA were similar to those in the 1995 NHS. It is difficult to make direct comparisons given the nationally used age bands may hide any variations in health.

Evidence-based physical activity recommendations specifically for older Australians have been introduced since these data were collected [21]. Their aim is to motivate older people to adopt and maintain a range of forms of physical activity in accordance with their capacity, goals and preferences. The recommendation concerning frequency and duration is in line with the original adult guidelines: at least 30 minutes of moderate-intensity activity on most days of the week. Comparing the prevalence rates reported here to this recommendation, we can see that there remains considerable scope to increase the proportion of older people who are physically active. Armstrong and colleagues [8] assessed the impact of recent physical activity campaigns on intention to be active. Older people were less likely than younger ones to intend to become active: $56.5 \%$ did not intend to become active, only $22.5 \%$ intended doing so in the next month and $20.9 \%$ in the next 6 months. However, older people were more likely to recall a generic physical activity campaign (around 60\%) (Active Australia) and about 24\% recalled a more specific campaign for older people [22]. This indicates that media campaigns may be a particularly effective means to promote physical activity in older people. As the physical activity recommendations for older people remain to be widely disseminated, there is the opportunity to assess their impact on physical activity in the coming years. However, it is recognised that recommendations alone do not change behaviour. The evidence emphasises the need for multifaceted approaches, including supportive physical and social environments for activity and attention to individual motivators for behaviour change, to promote and support physical activity among older people $[23,24]$. Australia benefits from the presence of a broad range of programs tailored for older people, such as the COTA Australia 'Living Longer, Living 
Figure 2: Comparison of log transformed means (95\% confidence intervals (Cls)) for time in minutes per week spent (a) walking, (b) in moderate activity and (c) in vigorous activity, Dynamic Analyses to Optimise Ageing (DYNOPTA) and National Health Survey (NHS) 2001, men and women.

(a)

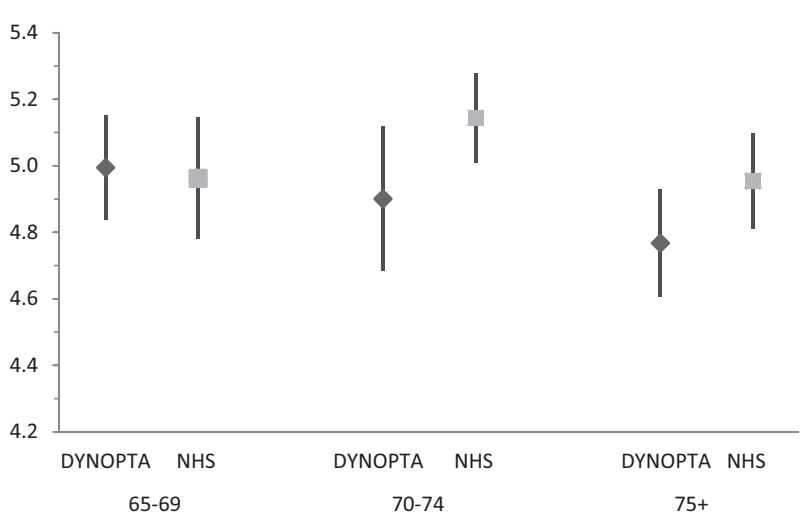

(b)

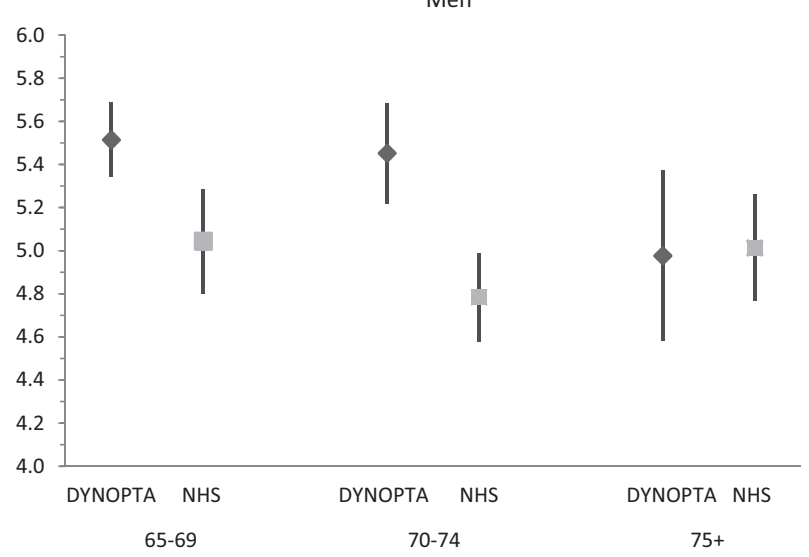

(c)

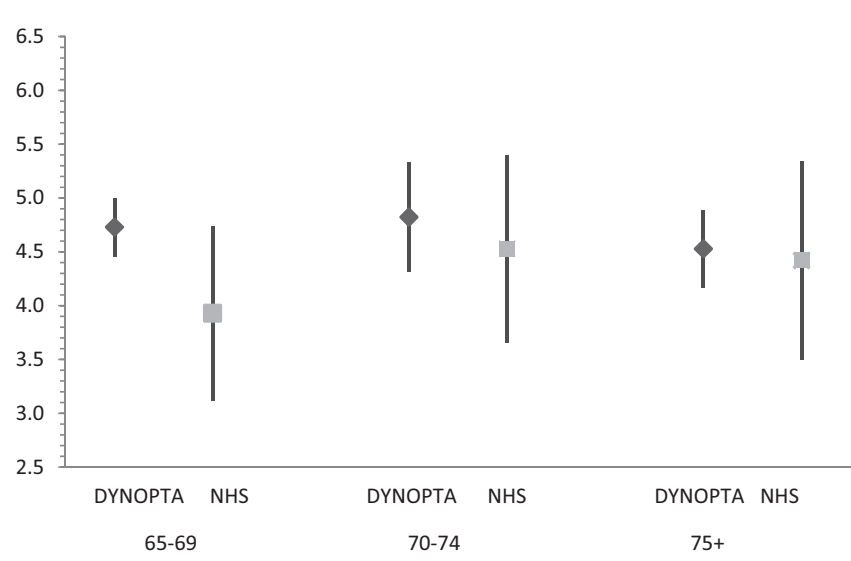

Stronger' program [25]. The challenge remains to extend the reach of these programs across older people.

Use of the pooled dataset has some limitations. Not every study contributed every physical activity variable data to the harmonised dataset. As not all contributing datasets were
Women

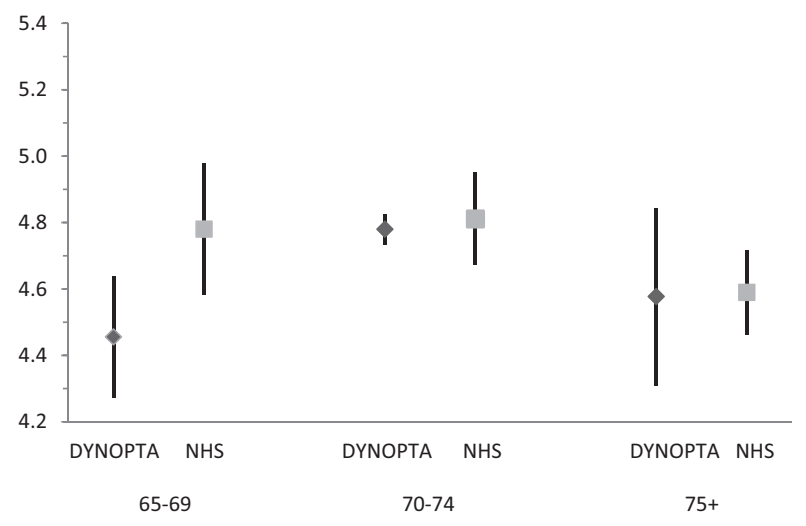

Women

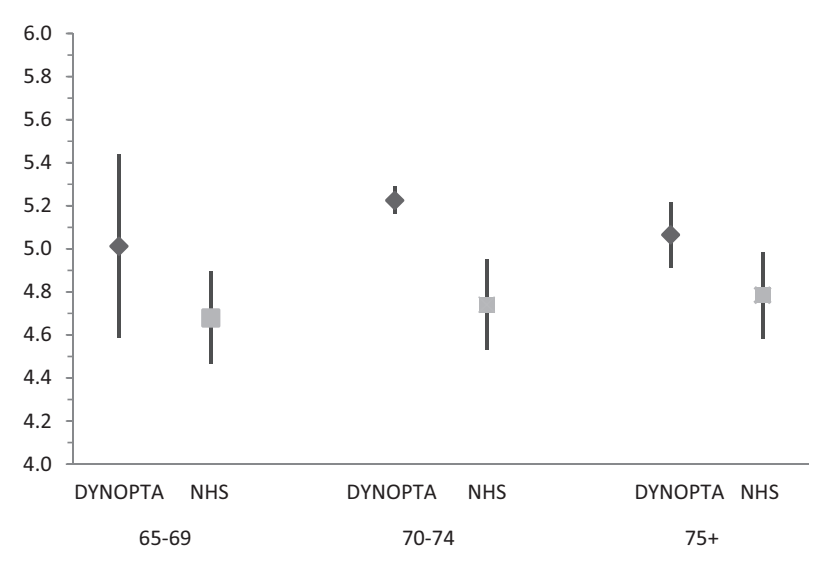

Women

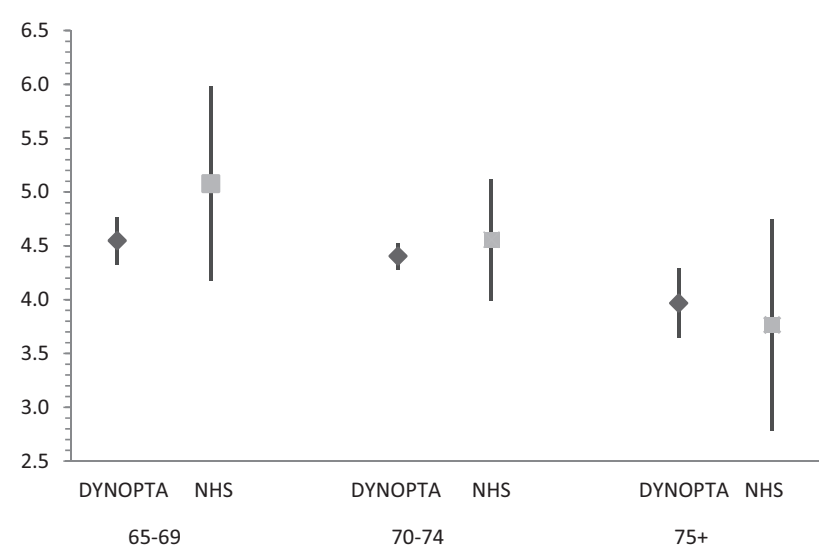

nationally representative, the sample needed to be weighted to produce population estimates. The physical activity data were obtained by self-report; while this is accepted method, particularly in large-scale studies, it can create reporting bias. There was variability between studies in the sampling methods used, the inclusion and exclusion criteria, and 
modes of survey administration and this potential for heterogeneity had to be tested and adjusted for. The largest proportion of the sample examined in this paper was of older women in their seventies. This and the large proportion that performed no activity created skewed data and raises queries about the representativeness of the data from those who were active. It was not possible to analyse the activity patterns of the 'oldest old' (85+ years) because of their relatively small numbers in the current dataset.

A strength of the harmonised data was the reference to a 1-week recall period for physical activity. The NHS refer to the past fortnight. Although the NHS results were divided by two to produce a weekly value, the activity may not have been evenly spread across the 2 weeks. Bursts of activity, where 1 week is spent in sedentary behaviour and the next in exercise may not impart the same protective health benefits as more regular activity. The physical activity patterns of over 10 thousand people could be examined using the DYNOPTA data. Crucially, the DYNOPTA data enabled detailed exploration of the physical activity patterns of those aged 75 years and over.

\section{Conclusions}

Using aggregated data for the 'old old' (75+ years) may mask differences in the two generations this group comprises. The harmonised data, while reflecting an age-related decline in physical activity, indicate that this is more prominent among men than women and when people reach their ninth decade, that is, among those aged 80 and over. Although our data are a decade old, the ABS statistics for the most recent NHS surveys 2004-2005 [26] and 2007-2008 [27] indicate that the prevalence of sedentary behaviour among older Australians is increasing. There is clearly a great need to develop and implement policy and programs to promote greater rates of physical activity in our older community.

\section{Acknowledgements}

The data on which this research is based were drawn from several Australian longitudinal studies including: the Australian Longitudinal Study of Ageing, the ALSWH, AusDiab, the $B M E S$, the Canberra Longitudinal Study of Ageing, the Household, Income and Labour Dynamics in Australia study, the Melbourne Longitudinal Studies on Healthy Ageing, the Personality And Total Health Through Life Study and the SOPS. These studies were pooled and harmonised for the DYNOPTA project. DYNOPTA was funded by a National Health o Medical Research Council grant (\# 410215). All studies would like to thank the participants for volunteering their time to be involved in the respective studies. Details of all studies contributing data to DYNOPTA, including individual study leaders and funding sources, are available on the DYNOPTA website (http:/ldynopta.anu.edu.au). The findings and views reported in this paper are those of the authors and not those of the original studies or their respective funding agencies. The authors would like to thank David Steele, University of Wollongong, for his statistical advice.

\section{Key Points}

- The absence - and aggregation - of data has limited our understanding of the physical activity patterns of older people.

- The current findings from DYNOPTA highlight that on average older people continue to walk for exercise until around the age of 80 .

- Most older people do no moderate or vigorous activity.

- Physical activity levels remain to be increased among older people of all ages.

\section{References}

1 Australian Bureau of Statistics. Population Projections Australia. ABS, ed. Canberra: ABS, 2008.

2 World Health Organisation. Active Ageing: Towards Age-Friendly Primary Health Care. Geneva: WHO, 2004.

3 Kendig H, Browning C, Pedlow R, Wells Y, Thomas S. Health, social and lifestyle factors in entry to residential aged care: An Australian longitudinal analysis. Age and Ageing 2010; 39: 342-349.

4 Sedentary Behaviour Research Network. Standardized use of the terms 'sedentary' and 'sedentary behaviours'. Applied Physiology Nutrition and Metabolism 2012; 37: 540-542.

5 Sims J, Hill K, Hunt S et al. National Physical Activity Recommendations for Older Australians: Discussion Document. Canberra: Australian Government Department of Health and Ageing, 2006.

6 Bijnen FCH, Feskens EJM, Caspersen CJ, Mosterd WL, Kromhout D. Age, period, and cohort effects on physical activity among elderly men during 10 years of follow-up: The Zutphen Elderly Study. Journals of Gerontology Series B, Psychological Sciences and Social Sciences 1998; 53: M235M241.

7 Australian Sports Commission. Active Australia National Physical Activity Survey, 1997. Canberra: Australian Sports Commission, 1998.

8 Armstrong T, Bauman A, Davies J. Physical Activity Patterns of Australian Adults: Results of the 1999 National Physical Activity Survey. Canberra: Australian Institute of Health and Welfare, 2000.

9 Bauman A. Health benefits of physical activity for older adults - Epidemiological approaches to the evidence. In: Morris M, Schoo A, eds. Optimizing Exercise and Physical Activity in Older People. Edinburgh: Butterman Heinemann, 2004: 1-25.

10 Taylor WC, Baranowski T, Young DR. Physical activity interventions in low-income, ethnic minority, and populations with disability. American Journal of Preventive Medicine 1998; 15: 334-343.

11 New South Wales Health. The Active Australia/International Year of Older Persons Public Education Campaign to Promote Physical Activity among Older People: NSW Evaluation Report. Sydney: NSW Health Department, 2000.

12 Anstey K, Byles J, Luszcz M et al. Cohort profile: The Dynamic Analyses to Optimize Ageing (DYNOPTA) project. International Journal of Epidemiology 2010; 39: 44-51.

13 Department of Health and Ageing. An Active Way to Better Health: National Physical Activity Guidelines for Adults. Canberra, ACT: DoHA, 2005.

14 Australian Bureau of Statistics A. National Health Survey: Users' Guide, 2001. Canberra: ABS, 2001.

15 Craig CL, Marshall AL, Sjostrom M et al. International Physical Activity Questionnaire: 12-country reliability and validity. Medicine and Science in Sports and Exercise 2003; 35: 1381-1395.

16 Byles J, Gibson R, Humphreys M. Age associated decline in physical mobility: Trends and transitions. Australasian Journal on Ageing 2009; 28 (Suppl. 2): 47.

17 Anstey K, Bielak A, Birrell C et al. Understanding ageing in older Australians: The contribution of the Dynamic Analyses to Optimise Ageing (DYNOPTA) project to the evidence base and policy. Australasian Journal on Ageing 2011; 30 (Suppl. 2): 24-31.

18 Burns RA, Butterworth P, Windsor T, Luszcz MA, Ross LA, Anstey KJ. Deriving prevalence estimates of depressive symptoms throughout 
middle and old age in those living in the community. International Psychogeriatrics 2012; 24: 503-511.

19 Anstey KJ, Burns RA, Birrell CL, Steel D, Kiely KM, Luszcz MA. Estimates of probable dementia prevalence from population-based surveys compared with dementia prevalence estimates based on meta-analyses. BMC Neurology 2010; 10: 62.

20 Bartsch L, Butterworth P, Byles J, Mitchell P, Shaw JKA. Examining the SF36 in an older population: Analysis of data from the Dynamic Analyses to Optimise Ageing (DYNOPTA) project. Quality of Life Research 2011; 20: $1227-1236$

21 Sims J, Hill K, Hunt S, Haralambous B. Physical activity recommendations for older Australians. Australasian Journal on Ageing 2010; 29: 81-87.

22 Bauman A, Armstrong $\mathrm{T}$, Davies $\mathrm{J}$ et al. Trends in physical activity participation and the impact of integrated campaigns among Australian adults, 1997-99. Australian and New Zealand Journal of Public Health 2003; 27: 76-79.

23 King AC. Interventions to promote physical activity by older adults. Journals of Gerontology: Series A 2001; 56: 36-46.

24 Kahn EB, Ramsey LT, Brownson R et al. The effectiveness of interventions to increase physical activity: A systematic review. American Journal of Preventive Medicine 2002; 22: 73-107.

25 Council on the Ageing. Living Longer Living Stronger Program. 2012. [Cited 3 July 2012.] Available from URL: http://www.Illswa.org.au/

26 Australian Bureau of Statistics A. National Health Survey: Summary of Results 2004-2005. Canberra: Australian Bureau of Statistics, 2006.

27 Australian Bureau of Statistics. National Health Survey; Summary of Results, 2007-2008 (Reissue). Canberra: ABS, 2009. 